\title{
PSYCHOPATHOLOGY AND THE THEORY OF PSYCHOPATHIC (GERMINAL) INHERITANCE.
}

\author{
By I. D. SUTTIE, Glasgow.
}

Is the psychological interpretation of a mental disease such as paranoia we are under the necessity of explaining the statistical fact of its high familial incidence. For the physico-chemical interpretation, however, the heritability of 'delusional insanity' presents no difficulty ; indeed, in the absence of any specific histopathological findings, any characteristic lesion or abnormality whatever, the evidence of its inheritance is the only empirical ground for assuming in this mental disease a structural basis determined otherwise than by function. Since there is physical but not psychical continuity between successive generations, it might be argued that structure alone is transmitted; so far as morbid inheritance is proved a significant factor in the etiology of the neuroses, to the same extent the psychological etiology will be invalidated.

In this way the question of inheritance becomes crucial for psychiatry. Those diseases which are fatally determined $a b$ ovo must have a purely 'organic' development (pathogenesis), must be studied and formulated 'physically'; effective treatment (if any such be imaginable) must follow the same lines, and it would appear that prophylaxis is limited to eugenic measures. If the germinal cause be necessary, but not in itself sufficient, then prophylaxis or treatment that preserves the individual without diminishing his fertility (or at least limiting it in certain directions) will defeat its own ends by disseminating the predisposition through the population. In so far as we attribute pathogenic efficiency to a germinal abnormality (always hypothetical), we limit the significance of 'trauma'. By solving the etiological problem in this manner we decrease the value and interest of the study of disease ; by emphasizing the importance of germinal determination (morbid), and hence of inherited structure, we assign a superficial rôle to psychological interpretations. This would not further our understanding of the phenomena of paranoia and the neuroses.

It should be noticed that those diseases with mental symptoms in which an hereditary transmission has been most clearly made out, are precisely those in which the organic basis is most obvious. 
The ideal of psychopathological interpretation is the formulation (from the standpoint of both experience and behaviour) of the nature and development of the morbid condition under its consideration, and the reference of abnormalities to traumata (in the widest sense) acting through the sense organs (i.e., by normal afferent channels) and not, by physical or chemical means, directly upon nerve tissue. Fundamental, endogenous abnormalities of disposition are subjectively unintelligible; they could not be appreciated by the patient or communicated to the doctor, since each interprets mind in terms of his own, and words exist only for trans-subjective meanings. The subjective evaluation of innate disposition is then impossible (except by exclusion); behaviour gives at best a rough quantitative indication of this ; and it is obvious that psychopathology cannot assimilate the 'individual' and the 'innate' into its causal sequence. It is concerned with abnormal modifications of thought and conduct due to abnormal experience, and its objective is to complete as far as possible this causal explanation, deducing therefrom rational prophylaxis and treatment.

So far as values and qualitative experience generally are concerned, we have no resources but the current psychological conceptions interpreted in terms of our own experience. By what other vehicle than terms of accepted meaning can the patient communicate to us his thought processes? He has no standard of comparison to enable him to appreciate his abnormality; even if he had a supernaturally acquired insight, he has no means of imparting to us an appreciation of any qualitative differences between his mind and ours. Only in so far as minds are similar can they develop (since development is a social process); only in so far as they are similar can they be scientifically studied and understood. Even could psychological analysis delve down (subjectively) to the bedrock of inherited tendencies, etc., it could never satisfactorily generalize these, could never make of them a scientific pathology.

For the psychopathologist, then, a patient is a potentially normal mind whose feelings, beliefs, attitudes, interests, and other reactions have been warped by abnormal experience. To an understanding of trauma and pathogenesis he looks for prophylaxis and therapy. The innate and the individual are an irresoluble residual which represent the limitations of his method. He is concerned to criticize the genetic interpretation put upon the familial incidence of mental diseases. For those diseases whose transmission is proved to be physiological he must abandon etiological research; where proof is wanting he must insist on the question remaining open.

I think it is sufficiently obvious that where transmission of any disease with mental symptoms is proved to take place through the 
mechanism of physical heredity, the physiological interpretation of that disease starts with a fundamental advantage-the certainty that its method is valid. On the other hand, in studying these diseases psychopathology must take account of a fact that is outside its 'universe of discourse'.

Here, then, in the mode of inheritance of any particular mental disease, is a criterion which will enable us to choose our line of research, which might save the psychopathologist a fruitless task. If the disease is inherited by physical channels, its fundamental cause and nature are physical. Psychological formulation is secondary and superficial ; psychotherapy at best a palliative; mental hygiene, as I say, a means of propagating unsound stock. Both theoretically and practically, then, it is a matter of fundamental importance to attain definite knowledge of the validity of the interpretation of the facts in terms of physical heredity, and whether there is any possible alternative interpretation.

Failing organic defects or stigmata whose correlation with innate defect of mind is completely established, the only positive proof of an organic transmission of mental characters would be a 'Mendelian' distribution among offspring. The noteworthy attempt of Davenport and Weeks to demonstrate this has been criticized by Mott himself, and we will here only remark that it is fully made out only in those diseases whose 'organic' basis is otherwise fully manifest. Unless such a 'specific pattern' is demonstrated, statistics can show only $a$ connection between cases, and it seems worth while to consider what alternative causal connection (to the germinal one) can exist. For the purposes of this inquiry we can accept the statistics of familial incidence without criticism, being merely concerned to show all possible causal connections between cases of mental disease, other than hereditary transmission and physical infection.

It is obvious that if trauma is here of any etiological importance, exposure to identical traumata must be a factor in certain 'bad family histories'. Unless the ontogenesis of mental disease be denied, it must be admitted that environmental causes themselves tend to produce a grouping of cases within certain families, in excess of theoretical expectation. That is to say, the incidence will not be 'random'; in the absence of all causal connection between cases, physical or psychical, statistics would still show a tendency to family grouping; by bearing on each generation in turn, such traumata as those arising from poverty and bodily weakness or deformity will tend to produce a succession of mental troubles within the family simulating inheritance.

For psychic characters other channels of transmission exist than the mechanism of physical inheritance. A child's imitation of 
mannerisms, standards, and judgements is notorious. Its suggestibility is not merely positive; even the simple organic appetites are profoundly modified by upbringing. Interests, ambitions, sense of value and 'proportion', 'common-sense', 'moral' sense, sociability (or at least attitude to and interest in one's fellows), habits of industry, occupational 'bent', skill and capacity, tastes, enjoyments, hobbies, amusements, to say nothing of the capacity for clear and independent thinking and for controlling conduct in accordance with decisions so arrived at-all these are mainly acquired. Yet these practically form character and disposition; $\dot{a}$ healthy balance in these is mental health. Of course it will be argued that the 'predisposition' interferes with the establishment of these mental qualities, etc. This must, however, be proved in general, and not merely assumed in regard to those cases which eventuate in psychoses, etc. It may be accepted, however, as certain that upbringing, as distinct from trauma, has much to do with mental stability or psychopathic predisposition, quite independently of our opinion with regard to the Freudian etiology, the psychic traumata of infancy, etc., and the pathogenic potentialities of certain family relationships.

All these considerations indicate the importance of such ontogenetic factors as, e.g., parental disagreement, irritability, or overindulgence; the presence of a neurotic, psychotic, or defective member in the child's immediate environment. The family 'tradition' or 'atmosphere', its interests and amusements; its resources for occupying and developing rather than repressing the growing mind; play, games, books, companionships, social ideals and customs-all have a hygienic or pathogenic effect which is limited to the family and therefore expresses itself in statistics in a way indistinguishabie from (non-Mendelian) inheritance. That is to say, that, whatever their influence for good or evil, these factors affect families as wholes (to a large extent), thus making character, tastes, disposition, ability, temperament, and mental stability to some extent acquired family charasters. Only the specific pattern of a Mendelian distribution (by indicating a chromosomic determinant) will demonstrate unequivocally a physical rather than this psychic transmission. Hence the significance of the work of Davenport and Weeks. Failing the proof of this, the alternative is a suspension of judgement pending an evaluation of the psychic factors.

I have attempted to show that the neuropathological theory of mental disease is associated with the hypothesis of its hereditary transmission. I think even that the two lend each other 'moral' as well as logical support. I have also indicated that the crucial test of the ultimate validity of psychopathological interpretation is its ability to account for high familial incidence of the disease-form to 
which it is applied. If one might hazard a tentative judgement on these lines while presuming an agreement upon the evidence, one might say that as certain mental syndromes have no typical or integrated psychic aspect, but have, on the other hand, a demonstrably organic basis, we must infer that any hereditary relation is physical, and mutatis mutandis where no neural lesion or abnormality is discoverable. Thus mental defect, as we know, is heritable; the psychoses are questionably so, with the exception of paranoia, which with the minor and anomalous neuroses are probably acquired. 